\title{
Die SimplyScience Stiftung
}

\author{
Thomas Flüeler*
}

\begin{abstract}
SimplyScience is an internet platform for 8 to 18 year olds and their parents and teachers, created by the scienceindustries in 2008. scienceindustries represents the chemical, pharma and biotech industries and is active in the Swiss Federal Department of Economic Affairs, Education and Research's programme 'Promotion of Science, Mathematics and Technology', the so-called 'MINT' subjects. SimplyScience presents articles, videos and podcasts on diverse scientific subjects and has the objective of interesting children and adolescents for science and technology matters and thereby encouraging them to consider a career in one of the MINT subjects.
\end{abstract}

Keywords: MINT · SimplyScience · Young people

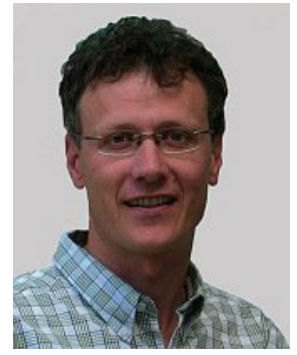

Thomas Flüeler is Managing Director of the SimplyScience Foundation, an initiative of scienceindustries to promote the interest of young people in science and technology. He holds an ETH master degree in biochemistry and is a former headmaster, currently still teaching biology and chemistry at the Kantonsschule Sargans.

\section{Anfänge mit SimplyScience.ch}

SimplyScience wurde 2008 von scienceindustries als Internetplattform für 12-16-Jährige ins Leben gerufen. Diese Initiative des Wirtschaftsverbands Chemie Pharma Biotech wird seither von zahlreichen Partnern aus Wirtschaft, Wissenschaft, Bildung und Medien unterstützt und ist Teil des Massnahmenplans „Nachwuchsförderung von Naturwissenschaften, Mathematik und Technik" des Eidgenössischen Departements für Wirtschaft, Bildung und Forschung. SimplyScience.ch präsentiert Artikel, Videos und Podcasts zu unterschiedlichen naturwissenschaftlichen Themen, insbesondere im Bereich Chemie und Biologie, und bezweckt, das Verständnis von Jugendlichen für naturwissenschaft-

\footnotetext{
*Correspondence: T. Flüeler, Geschäftsführer SimplyScience Stiftung

Nordstrasse 15

$\mathrm{CH}-8021$ Zurich

E-mail: thomas.flueeler@simplyscience.ch

www.simplyscience.ch
}

lich-technische Fragen, die sogenannten MINT-Fächer, zu fördern und sie dabei für Ausbildungs- und Laufbahnmöglichkeiten in Wissenschaft und Wirtschaft zu begeistern. Gleichzeitig sollen ansprechend aufbereitete Informationen auch in der breiten Bevölkerung zu einer erhöhten Akzeptanz der Wissenschaften im Allgemeinen und der Chemie im Speziellen führen.

\section{Entwicklung in den letzten zehn Jahren}

Die grundlegenden Zielsetzungen haben sich seit der Lancierung von SimplyScience nicht verändert. Erfreulicherweise konnten aber Fokus und Reichweite der Initiative in den letzten knapp zehn Jahren deutlich vergrössert werden. Zwei Jahre nach der Lancierung der Internetplattform wurde SimplyScience im Jahr 2010 in eine unabhängige Stiftung überführt. Die Website wurde 2012 visuell völlig neu gestaltet und inhaltlich um einen französischen Teil erweitert, damit SimplyScience als Medium sowohl in der Deutsch- als auch in der Westschweiz verankert werden konnte. Zudem wurde die Zielgruppe altersmässig verbreitert, so dass sich heute Kinder und Jugendliche von 8 bis18 Jahren ebenso wie ihre Familien und Lehrpersonen von den Inhalten auf SimplyScience.ch angesprochen fühlen. Insgesamt erzielt die Website aktuell über 1,5 Millionen Seitenaufrufe (bei mehr als einer Million Seitenbesuchen) jährlich.

\section{Inhalte und Zielsetzungen}

Texte und Bildergalerien $\mathrm{zu}$ den Themen werden vom Redaktionsteam gezielt für Kinder und Jugendliche ausgewählt, fundiert recherchiert und verständlich aufbereitet. Die Inhalte werden gerne auch von Lehrpersonen im Unterricht eingesetzt. Nichts weckt die Neugierde an
Naturwissenschaften jedoch nachhaltiger als die eigene, aktive Erfahrung mit den Phänomenen und Gesetzen, die unseren Alltag prägen. Dies gilt insbesondere in den Bereichen der Chemie und Biologie. Auf SimplyScience findet sich deshalb inzwischen eine umfangreiche Sammlung von Anleitungen für einfache Experimente. Sie laden dazu ein, Phänomene zu hinterfragen, eine forschende Herangehensweise zu entwickeln und in die naturwissenschaftliche Denkweise einzutauchen. Dazu braucht es weder ausgefallene Reagenzien noch übermässigen Aufwand: Alle Versuche sind mit ungefährlichen Materialien und Stoffen durchführbar, die ohnehin im Haushalt vorhanden oder einfach zu beschaffen sind. So wird der Zugang zum Experimentieren möglichst niederschwellig gehalten, und naturwissenschaftliche Themen werden in einen direkten Bezug zum Alltag gebracht. Erstaunliche Ergebnisse und AhaErlebnisse, aber auch Missgeschicke und die Suche nach Verbesserungen und anderen Lösungswegen tragen dazu bei, dass Kinder ihre natürliche Faszination für Experimente behalten und ein allgemeines Interesse an naturwissenschaftlichen Arbeitsweisen entwickeln.

Jugendliche, welche diese Faszination schon früh erlebt haben und beim aktiven Experimentieren unterstützt wurden, sind auch in der Schule leichter für Fächer wie Chemie, Physik oder Biologie zu begeistern und ziehen eher eine Ausbildung in diesen Gebieten in Betracht. Dieser Aspekt ist für die Initianten von SimplyScience von grosser Bedeutung, da qualifizierte Fachkräfte im Bereich von Naturwissenschaften heute mangeln und der Bedarf in Zukunft noch steigen wird. Nicht zu unterschätzen ist jedoch auch die gesellschaftliche Bedeutung von Jugendlichen mit einem geschärften Blick und soliden Grundverständnis für naturwissenschaftliche Zusammenhänge sowie der Fähigkeit, kritische Fragen zu stellen und Probleme lösen zu können. Diese Eigenschaften sind in nahezu je- 
dem Berufsfeld von Nutzen und auch bei gesellschaftlichen und politischen Entscheidungen unabdingbar.

\section{Öffentlichkeitsarbeit und Partnerschaften}

SimplyScience engagiert sich dementsprechend dafür, dass Kinder jeglicher Herkunft Gelegenheit zu motivierenden Erlebnissen mit Naturwissenschaft und Technik erhalten. Die Stiftung arbeitet mit zahlreichen Partnern zusammen, die solche Erlebnisse ermöglichen, und stellt ihr Angebot regelmässig an Ausstellungen und anderen Öffentlichkeitsveranstaltungen für Kinder, Jugendliche und ganze Familien vor (Figur. 1).

Wichtiger Bestandteil ihrer Arbeit ist auch die Zusammenarbeit mit Schulen. Die SimplyScience Stiftung veranstaltet beispielsweise Wettbewerbe zu naturwissenschaftlichen Themen für Schulklassen, darunter den zweijährlichen Wettbewerb "Science on the Move“. Dieser Laborwettbewerb richtet sich an Gymnasialklassen aus der ganzen Schweiz und wird von der F. Hoffmann-La Roche AG unterstützt. Die erfolgreiche Teilnahme am Wettbewerb verlangt grosses Engagement von allen Schülerinnen und Schülern der Klasse, denn die Aufgabenstellung legt nicht nur Gewicht auf wissenschaftliches Denken und Laborfertigkeiten, sondern setzt ebenso Schwerpunkte bei Teamfähigkeit und Kreativität. Als Belohnung winkt der jeweiligen Siegerklasse eine Science Week in London (siehe Figur 1 in den Artikel von M. Sennhauser in diesen Heft $\left.^{[1]}\right)$, bei der ein reichhaltiges und lehrreiches Programm mit Besuchen verschiedener Wissenschaftsmuseen und Ausstellungen in London sowie 'Peer to Peer'-Begegnungen sowie Workshops mit englischen Studenten geboten werden.

Des Weiteren ist die SimplyScience Stiftung Herausgeberin von Lehr- und Experimentiermaterialien, welche Pädagoginnen und Pädagogen beim naturwissenschaftlichen Unterricht im Sinne des Lehrplans 21 unterstützen. Der Austausch zwischen Lehrpersonen wird in Weiterbildungskursen gefördert, die auch Gelegenheit bieten, verschiedene Methoden der Wissensvermittlung und des aktiven Experimentierens mit Schülerinnen und Schülern zu diskutieren.

\section{Lehrmittel}

Eines der ersten Lehrmittel, welches die SimplyScience Stiftung anregte und mitgestaltete, war der SimplyNanoExperimentierkoffer für die Sekundarstufe
Figur 1. Kinder beim Experimentieren und Entdecken am Stand von SimplyScience bei der tunSolothurn 2016, einer Erlebnisschau mit dem Ziel, Kinder und Jugendliche für Technik und Naturwissenschaften (tun) zu begeistern.



I. Er ermöglicht eine Veranschaulichung der Dimensionen und eine Einführung in die Grundlagen der Nanotechnologie und ist so beliebt, dass inzwischen der weiterführende Koffer SimplyNano II aufgelegt wurde (Figur 2). Dieser stellt Anleitungen und Materialien für 32 weitere Schülerexperimente im Bereich der angewandten Nanotechnologie zur Verfügung. Beide Koffer wurden im Auftrag der SimplyScience Stiftung von der Innovationsgesellschaft $\mathrm{mbH}$ entwickelt; ihre Produktion sowie die für Lehrpersonen kostenlosen Weiterbildungskurse werden neben SimplyScience von zahlreichen weiteren Sponsoren unterstützt.

Ebenfalls bereits in zweiter Auflage erschien in Zusammenarbeit mit Interpharma das spannende kombinatorische ChemieKartenspiel ChemiX, das bei Schülerinnen und Schülern ab dem 8. Schuljahr spielerisch das Interesse an chemischen Verbindungen und ihren Eigenschaften weckt. Das Materialpaket „Stoffe trennen und verbinden", vom Verein explore-it im Auftrag der SimplyScience Stiftung entwickelt, erfreut sich an Primarschulen grosser Beliebtheit. Für dieselbe Schulstufe stehen auf der SimplyScience-Website unter dem Namen ,SimplyHuman“ Anleitungen und Experimentiervorschläge $\mathrm{zu}$ den Themen Auge sowie Muskeln/Skelett zum Download zur Verfügung. Das Lehrmittel wurde von der Pädagogischen Hochschule

Zürich entwickelt und von Novartis AG finanziell unterstützt; Fortbildungen für Lehrpersonen und die Konfektionierung von Materialboxen sind für 2018 geplant.

"Chemie für dich und mich" lautet der Titel einer Experimentierbox für die 5. und 6. Klasse der Primarschule, die von SimplyScience unter Beratung durch die Pädagogische Hochschule Thurgau entwickelt wurde. Sie enthält Unterrichtsmaterialien zum Thema Alltagschemie, beginnend mit einem Einführungsmodul und dem Modul „Zaubermittel Waschmittel“", und ist in den nächsten Jahren weiter ausbaubar.

Ende 2017 wurde das Lehrmittel „SimplyCooking“ mit der dazugehörigen, für Mobilgeräte optimierten Website unter www.simplycooking.ch veröffentlicht. Unterstützt durch Nestlé AG, die Schweizerische Gesellschaft für Ernährung sowie den Kanton St. Gallen wurde eine Sammlung von Rezepten rund um die Themen Eiweiss, Fett, Kohlenhydrate und Gelierungsmittel für die Schulküche aufbereitet und mit umfangreichen Informationen rund um die chemischphysikalischen Vorgänge bei unterschiedlichen Zubereitungsarten ergänzt.

Received: September 26, 2017

[1] M. Sennhauser, Chimia 2018, 72, 7.

Figur 2.

SimplyNano: Die Experimentierkoffer SimplyNano I und SimplyNano II ermöglichen eine praktische Einführung in die Grundlagen der Nanotechnologie.



